\title{
Variabilidad espacio-temporal de los eufáusidos frente a la costa de Concepción, Chile
}

\author{
Lorena Linacre* \& Sergio Palma \\ Escuela de Ciencias del Mar, Pontificia Universidad Católica de Valparaíso \\ Casilla 1020, Valparaíso, Chile \\ e-mail: llinacre@cicese.mx; spalma@ucv.cl
}

RESUMEN. Se analizó la distribución espacio-temporal de los eufáusidos colectados en cuatro cruceros estacionales realizados frente a la costa de Concepción en 1991. Se identificaron ocho especies, Euphausia mucronata, E. vallentini, Nyctiphanes simplex, Nematoscelis megalops, Nematobrachion flexipes, Stylocheiron longicorne, S. affine y S. suhmii. La mayoría de las especies había sido registrada en aguas del Pacífico suroriental, a excepción de S. suhmii, que se distribuye comúnmente en el Pacífico central. La presencia de $S$. suhmii constituye su primer registro en el Sistema de la Corriente de Humboldt. Del total de especies, E. mucronata (76\%) y N. simplex (23\%) fueron las más abundantes y frecuentes. Los eufáusidos se registraron en todos los periodos del año, aunque con fluctuaciones estacionales y espaciales asociadas a las características oceanográficas del área de estudio. Los máximos estacionales se determinaron en otoño y los mínimos en invierno, presentando diferencias significativas en su distribución espacial. Se encontró una relación inversa entre la abundancia de E. mucronata y la concentración de oxígeno disuelto, asociada a eventos de surgencia costera.

Palabras claves: eufáusidos, distribución, abundancia, surgencia, Concepción, Chile.

\section{Spatial and temporal variability of eufausiids off Concepción coast, Chile}

\begin{abstract}
Spatial and temporal distribution of euphausiids collected in four seasonal cruises of Concepción in 1991 was analyzed. Eight species of euphausiids were identified, Euphausia mucronata, E. vallentini, Nyctiphanes simplex, Nematoscelis megalops, Nematobrachion flexipes, Stylocheiron longicorne, S. affine and S. suhmii. Most species have been previously recorded in the Southeastern Pacific Ocean, with exception of $S$. suhmii, that is commonly distributed in the Central Pacific Ocean. The presence of $S$. suhmii constitutes the first record for the Humboldt Current System. The most abundant and frequent species were E. mucronata $(76 \%)$ and $N$. simplex $(23 \%)$. The euphausiids were collected in different seasons of the year, even though they show seasonal and spatial fluctuations associated to the oceanographic conditions. The seasonal maximum was determinate in autumn and the minimum in winter. Significant differences between the different oceanographic sections were found. Besides, an inverse relationship between the abundance of E. mucronata and the dissolved oxygen concentration was found, associated to coastal upwelling events.
\end{abstract}

Key words: euphausiids, distribution, abundance, upwelling, Concepción, Chile.

\section{INTRODUCCIÓN}

Los eufáusidos son uno de los grupos dominantes del zooplancton marino, especialmente sobre la pla- taforma continental y en áreas de alta productividad, donde se caracterizan por su elevada abundan-

\footnotetext{
* Departamento de Ecología, Centro de Investigación Científica de Educación Superior de Ensenada, CICESE. Apartado Postal 2732, Ensenada, Baja California 22830, México.
} 
cia y baja diversidad (Gibbons et al., 1999). Varias especies son indicadoras de masas de aguas y son fuente alimenticia importante para numerosos recursos marinos, constituyendo un enlace entre la producción fitoplanctónica y los niveles superiores (Antezana, 1978). Algunas especies pueden formar concentraciones comparables en número y biomasa a las de copépodos, e incluso los superan en ciertas épocas del año y/o en determinadas regiones (Antezana \& Brinton, 1981). Así por ejemplo, en aguas de la Corriente de California la biomasa en peso húmedo de los eufáusidos varía de un $50 \%$ de la biomasa total en primavera a un 10-30\% el resto del año (Brinton \& Reid, 1986). Mientras que en el Sistema de la Corriente de Humboldt (SCH), la especie dominante Euphausia mucronata que se distribuye a los $10-39^{\circ} \mathrm{S}$, tiene una biomasa media equivalente al $46 \%$ de la biomasa zooplanctónica total, con variaciones latitudinales que fluctúan del 29 a 91\% (Antezana, 1978, 2002a).

El área costera frente a Concepción, como parte del SCH, se encuentra bajo la influencia de vientos anticiclónicos asociados al centro de alta presión del Pacífico suroriental, con vientos predominantes del $\mathrm{N}$ en invierno y del S-SW en primavera-verano, que inducen frecuentes e intensos eventos de surgencia costera, particularmente en torno a punta Lavapié, y un patrón de circulación caracterizado por una corriente subsuperficial hacia el polo (Arcos et al., 1987; Peterson et al., 1988; Sobarzo et al., 1993). Las estimaciones del transporte de Ekman indican que las aguas frías superficiales provienen de 150$200 \mathrm{~m}$ de profundidad (Agua Ecuatorial Subsuperficial, AESS), en escalas de 2 a 7 días (Arcos et al., 1987). En periodos de surgencia activa, ascienden a las capas superficiales aguas de hasta $11^{\circ} \mathrm{C}$ que cubren toda la plataforma, mientras que durante eventos leves, las aguas tienen mínimos de $13^{\circ} \mathrm{C}$ (Cáceres \& Arcos, 1991). Estos centros de surgencia costera son favorecidos por la topografía de la plataforma continental y la línea de costa. Asociados a estos eventos, se pueden encontrar frentes y remolinos (Djurfeldt, 1989; Cáceres \& Arcos, 1991; Mesías et al., 2001), que pueden actuar como mecanismos de retención de organismos.

Además, las aguas de la plataforma continental adyacente a la bahía de Concepción también son afectadas por estos eventos, donde la recirculación juega un rol importante en la productividad. Durante la surgencia activa, las aguas superficiales de la bahía se desplazan al NW, con el subsecuente flujo compensatorio por el fondo de AESS proveniente de la plataforma adyacente, que por su alto contenido de nutrientes favorece la concentración de clorofila $a$ en la bahía (Mesías \& Salinas, 1986; Arcos et al., 1987; Ahumada et al., 1983, 1991). Asimismo, con un tiempo de residencia de 2 a 3 días, durante la relajación de la surgencia, se genera un aporte de aguas ricas en clorofila $a$ hacia fuera de la bahía (Ahumada et al., 1991). Esta alta biomasa fitoplanctónica es capaz de sustentar altas concentraciones de zooplancton, donde una fracción importante es transportada y dispersada hacia aguas adyacentes (Ahumada et al., 1991).

Diversos estudios muestran que los parámetros ambientales juegan un rol importante en la distribución de las comunidades planctónicas, especialmente sobre sus fluctuaciones latitudinales de abundancia. En los márgenes orientales de los océanos, los procesos de surgencia y la circulación determinan la permanencia espacio-temporal y estructura de las poblaciones de eufáusidos (Barange \& Pillar, 1992; Gibbons et al., 1995). Asimismo, en algunos sistemas, se ha mencionado que un comportamiento migratorio vertical en combinación con los patrones de circulación constituiría un mecanismo eficiente en la permanencia y desarrollo de algunas especies (Pillar \& Stuart, 1988; Pillar et al., 1989; Barange \& Pillar, 1992).

En aguas costeras frente a Concepción, esta conjugación entre los factores ambientales y el comportamiento poblacional, ya ha sido descrita para algunos zooplancteres. Tal es el caso, de las intrusiones del copépodo oceánico Rhincalanus nasutus residente en la Capa de Mínimo Oxígeno, CMO $\left(<1 \mathrm{ml} \mathrm{O}_{2} \cdot \mathrm{L}^{-1}\right)$, causadas por advección costera del AESS durante épocas de surgencia (Castro et al., 1993). Por esta razón, la zona costera de Concepción, se caracteriza no solo por su alta producción primaria (Ahumada et al., 1991), sino también por la estructura vertical de corrientes con diferentes direcciones, que dependiendo de las capacidades de desplazamiento vertical u horizontal de los organismos, puede ser utilizada como un mecanismo eficiente de transporte y retención por algunas especies.

En consecuencia, dado el elevado dinamismo ambiental que posee la zona costera de Concepción, afectada frecuentemente por eventos de surgencia, se analizará la variabilidad espacio-temporal de la composición, abundancia y distribución de los eufáusidos, así como el efecto de la CMO sobre la abundancia de Euphausia mucronata, que es el eufáusido dominante frente a la costa chilena. 


\section{MATERIALES Y MÉTODOS}

Las muestras de zooplancton se obtuvieron en cuatro cruceros oceanográficos estacionales efectuados en otoño (4 al 11 de abril), invierno (24 al 29 de junio), mediados de primavera (2 al 5 de noviembre) y fines de primavera (10 al 13 de diciembre), frente a la costa de Concepción ( $\left.36^{\circ} 06^{\prime}-37^{\circ} 00^{\prime} \mathrm{S}\right)$. En cada crucero se distribuyeron 15 estaciones oceanográficas en tres secciones perpendiculares a la costa, separadas entre sí por alrededor de $10 \mathrm{mn}$ (Fig. 1).

Las colectas de zooplancton se realizaron mediante pescas oblicuas diurnas y nocturnas realizadas desde $200 \mathrm{~m}$ a superficie, o desde cerca del fondo en las estaciones más someras, con redes Bongo de $60 \mathrm{~cm}$ de diámetro de boca y 335 micras de malla. Las redes estuvieron provistas de flujómetros para la estimación del volumen de agua filtrada. Las muestras se fijaron con una solución de agua de mar con formalina al $5 \%$ neutralizada con tetraborato de sodio.

Dentro de la comunidad de eufáusidos, solo los juveniles y adultos se separaron, contaron e identificaron, considerando los caracteres morfológicos señalados para estos organismos (Mauchline \& Fisher, 1969; Gopalakrishnan, 1975; Antezana \& Brinton, 1981; Antezana et al., 1997; Gibbons et al., 1999).

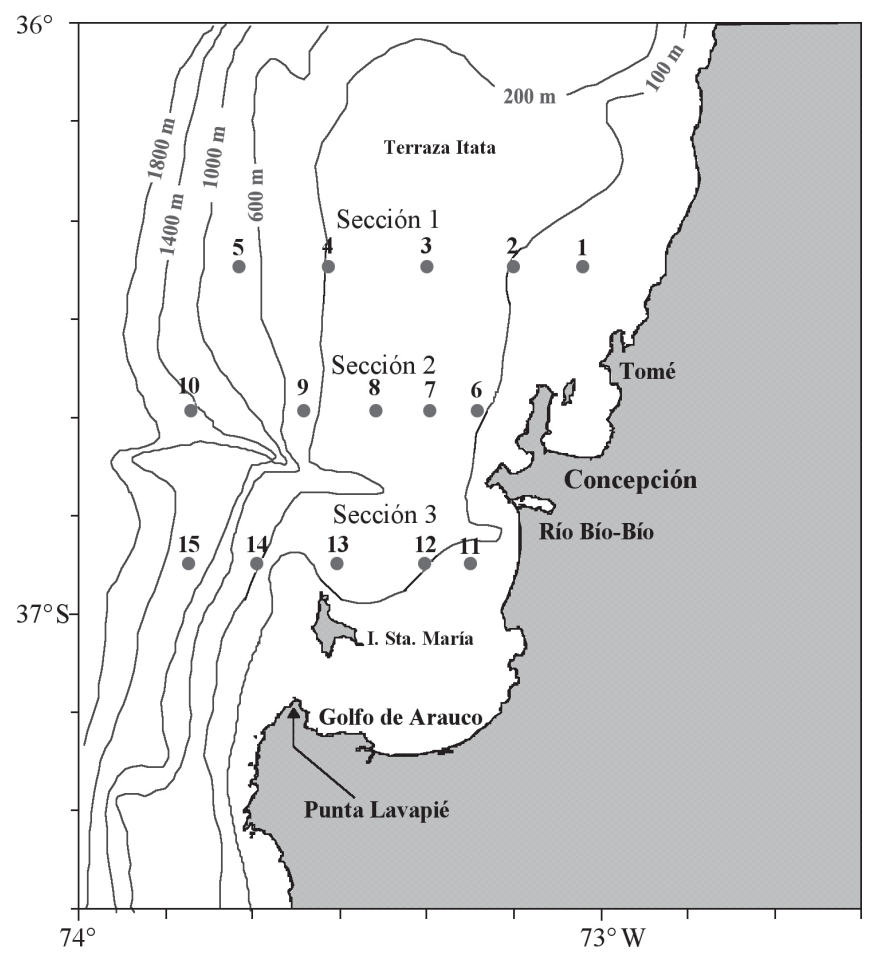

Figura 1. Ubicación de las estaciones de muestreo de zooplancton frente a la costa de Concepción.

Figure 1. Location of zooplankton sampling stations off Concepción coast.
La abundancia relativa se expresó en ind $1000 \mathrm{~m}^{-3} \mathrm{y}$ su representación gráfica se realizó según la escala métrica $\log ^{2}$ (Frontier, 1980). Para determinar la existencia de diferencias significativas en abundancia, se aplicó el análisis no-paramétrico de Kruskal-Wallis. Asimismo, para estimar el efecto de la hora de pesca en su abundancia, se realizó entre las pescas diurnas y nocturnas, la prueba no-paramétrica " $U$ " de Mann-Whitney.

Dado los antecedentes que relacionan la distribución de E. mucronata con la CMO (Antezana, 1978; Escribano et al., 2000; Ulloa et al., 2000), se efectuó un análisis de correlación Producto-Momento de Pearson entre la abundancia estacional de E. mucronata, transformada en $\ln (\mathrm{x}+1)$ para minimizar las diferencias entre los mínimos y máximos de abundancia, y los valores de oxígeno disuelto. Para el oxígeno disuelto se utilizó el promedio ponderado de los valores obtenidos en la columna de agua (0-200 m), utilizando la siguiente expresión:

$$
X_{i j}=\frac{\sum_{k=1} Z_{j k} * C_{i j k}}{\sum Z_{j k}}
$$

donde:

$\mathrm{X}_{\mathrm{ij}}$ : promedio ponderado del i-ésimo parámetro de la j-ésima estación

$\mathrm{Z}_{\mathrm{jk}}$ : k-ésima profundidad de la j-ésima estación

$\mathrm{C}_{\mathrm{ijk}}$ : valor del i-ésimo parámetro en la jésima estación a la k-ésima profundidad.

\section{RESULTADOS}

\section{Características oceanográficas}

Las características oceanográficas de cada crucero fueron descritas en detalle por Salinas (1992) y Silva (1993). En consecuencia, solo se indicarán los rasgos de mayor interés para este estudio, como son los eventos de surgencia que caracterizan esta zona. Los cruceros de abril, noviem- 
bre y diciembre, se caracterizaron por el ascenso de las isolíneas de los diferentes parámetros oceanográficos, indicando episodios de surgencia leve en abril y moderados en noviembre-diciembre (Silva, 1993). Consecuentemente, en las estaciones localizadas sobre la plataforma continental, se determinó la presencia del AESS en los primeros 50 $\mathrm{m}$ de profundidad, caracterizada por sus bajas concentraciones de oxígeno disuelto. En cambio, en junio la columna de agua se presentó bien oxigenada, con valores de salinidad de hasta 32 psu en superficie, debidos a la presencia de un cuerpo de agua resultante de la mezcla de Agua Subantártica (ASAA) y agua dulce del río Bío-Bío, que aumenta su caudal durante el invierno (Silva, 1993). En este mismo periodo, se registró un predominio de viento $\mathrm{N}$ en el área, mientras que en primavera fueron más frecuentes e intensos los vientos del S y SW (Salinas, 1992). El patrón de circulación se caracterizó por presentar corrientes paralelas a la costa, preferentemente en dirección sur durante la mayor parte del año, como asimismo flujos transversales a la costa que fueron variables en sentido e intensidad según la época del año (Salinas, 1992).

\section{Composición y abundancia}

Durante el periodo de estudio se determinó la presencia de ocho especies de eufáusidos: Euphausia mucronata Sars, 1883; E. vallentini Stebbing, 1900; Nyctiphanes simplex Hansen, 1911; Nematoscelis megalops Sars, 1883; Nematobrachion flexipes (Ortmann, 1893); Stylocheiron longicorne Sars, 1883; S. affine Hansen, 1910 y S. suhmii Sars, 1883.

Las especies más abundantes y frecuentes fueron E. mucronata (76\%) y N. simplex (23\%). Las especies restantes fueron ocasionales y constituyeron solo el $1 \%$ de los eufáusidos colectados. En abril se determinó el mayor número de especies y la mayor abundancia de organismos, mientras que en junio se registraron los valores más bajos de ambos parámetros (Tabla 1).

En el área de estudio, a excepción de noviembre, se determinó un fuerte incremento de abundancia de norte a sur, registrándose las mayores agregaciones en la zona sur, correspondiente al sector del golfo de Arauco (Sección 3). Sin embargo, los resultados de la prueba de Kruskal-Wallis, mostraron que solo en abril se determinaron diferencias significativas $(\mathrm{p}<0,05)$, entre las diferentes secciones de muestreo (Tabla 2), cuando el máximo otoñal fue cinco veces mayor que el de fines de primavera (Fig. 2).

\section{Distribución espacio-temporal}

Debido a la gran extensión de la plataforma continental en esta área (Fig. 1), la mayoría de las estaciones de muestreo se localizaron sobre la plataforma y consecuentemente, las pescas zooplanctónicas se efectuaron desde cerca del fondo a superficie. Así, a pesar que los eufáusidos realizan intensos despla-

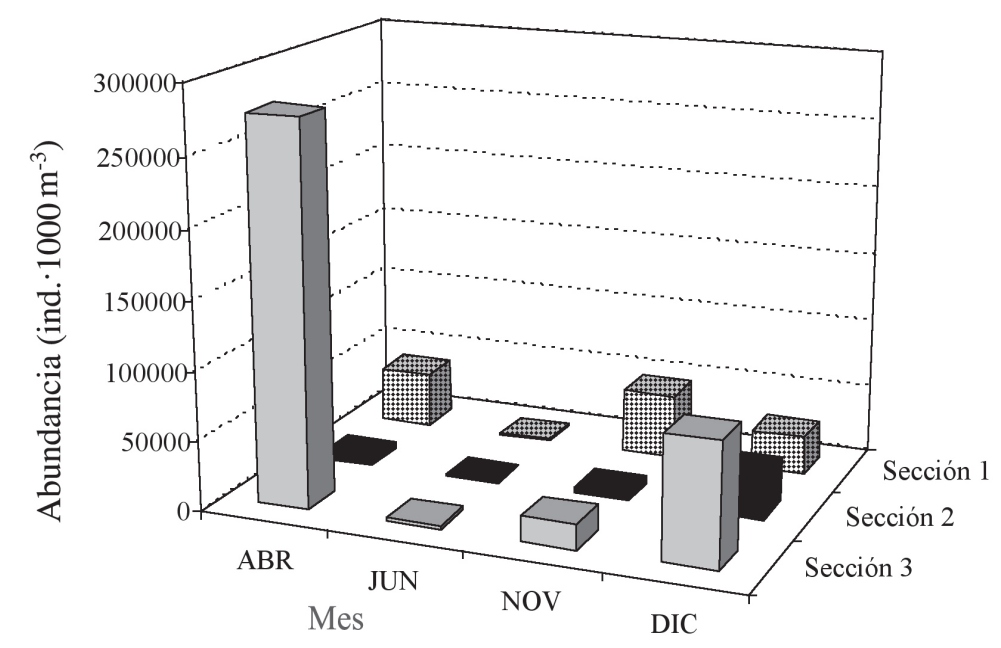

Figura 2. Abundancia de los eufáusidos en las secciones oceanográficas.

Figure 2. Abundance of euphausiids in the oceanographic sections. 
Tabla 1. Abundancia promedio de las especies de eufáusidos colectadas en los cruceros realizados frente a la costa de Concepción (individuos $1000 \mathrm{~m}^{-3}$ ).

Table 1. Mean abundance of euphausiid species collected in the cruises carried out off Concepción coast (individuals $1000 \mathrm{~m}^{-3}$ ).

\begin{tabular}{|lccccc|}
\hline Especie & Abril & Junio & $\begin{array}{c}\text { Crucero } \\
\text { Noviembre }\end{array}$ & Diciembre & Porcentaje \\
\hline Euphausia mucronata & 14.698 & 102 & 4.203 & 10.456 & 76,1 \\
Nyctiphanes simplex & 8.250 & 214 & 420 & 146 & 23,2 \\
Nematoscelis megalops & 21 & 1 & 24 & 113 & 0,4 \\
Euphausia vallentini & 13 & - & 40 & 8 & 0,2 \\
Stylocheiron affine & 20 & 1 & - & - & 0,1 \\
Nematobrachion flexipes & 3 & - & 1 & 7 & 0,0 \\
Stylocheiron longicorne & - & - & 0 & - & 0,0 \\
Stylocheiron suhmii & 1 & 3 & - & 0,0 \\
\hline
\end{tabular}

Tabla 2. Comparación de la abundancia total de las especies de eufáusidos entre las diferentes secciones oceanográficas efectuadas frente a la costa de Concepción. H: Prueba ANOVA por rangos de Kruskal-Wallis, N: número de estaciones de muestreo por crucero. Los valores significativos se indican en negrita $(\mathbf{p}<0,05)$.

Table 2. Comparison between the total abundance of euphausiid species and the oceanographic sections sampled off Concepción coast. H: Kruskal-Wallis ANOVA by ranks, N: number of sampling stations by cruise. Significant values are indicated in bold $(\mathrm{p}<\mathbf{0 . 0 5})$.

\begin{tabular}{|lccccc|}
\hline & & Suma de Rangos & H & p \\
& Sección 1 & Sección 2 & Sección 3 & $8,69(2, \mathrm{~N}=14)$ & $\mathbf{0 , 0 1 3 0}$ \\
Abril & 33 & 14 & 58 & $1,86(2, \mathrm{~N}=15)$ & 0,3946 \\
Junio & 33 & 36 & 51 & $4,86(2, \mathrm{~N}=15)$ & 0,0880 \\
Noviembre & 58 & 31 & 31 & $1,87(2, \mathrm{~N}=14)$ & 0,3923 \\
Diciembre & 29 & 29 & 47 & \\
\hline
\end{tabular}

zamientos nictimerales y que se verificó la existencia de diferencias significativas entre las capturas diurnas y nocturnas, para E. mucronata (Prueba de U de Mann-Whitney, $\mathrm{p}=0,0001$ ) y $N$. simplex (Prueba de U de Mann-Whitney, $\mathrm{p}=0,0000$ ), se puede considerar que la variabilidad en la abundancia diurno/nocturna fue mínima con respecto a la variabilidad estacional, ya que es muy probable que la mayor parte de estas poblaciones se encontraban en una columna de agua no mayor de $200 \mathrm{~m}$ de profundidad, independientemente de la hora de captura.

La especie más abundante y frecuente fue $E$. mucronata, la cual presentó una amplia distribución geográfica durante el año, con una diferencia estadísticamente significativa entre los periodos de muestreo (Prueba de Kruskal-Wallis, $\mathrm{p}=0$,0000). Las mayores densidades se registraron en abril, con un máximo de 69.542 ind $1000 \mathrm{~m}^{-3}$ en el sector más costero del golfo de Arauco, cuando las capturas fueron nocturnas. Al igual que las otras especies, los mínimos estacionales se registraron en junio, registrándose una recuperación de su abundancia en primavera, particularmente en diciembre (Fig. 3). Por su elevada abundancia, su patrón de distribución espacio-temporal fue representativo de la comunidad de eufáusidos (Tabla 1, Fig. 2).

$N$. simplex a pesar de ser un tercio menos abundante que E. mucronata, mostró una amplia cobertura geográfica y una diferencia significativa de abundancia entre los diferentes periodos analizados (Prueba de Kruskal-Wallis, p =0,0148). Sus máximos también se registraron en abril, con un máximo de 85.229 ind $\cdot 1000 \mathrm{~m}^{-3}$ en la estación 11 durante la noche (Fig. 4). Los restantes períodos del año fue mucho más escasa y a diferencia de E. mucronata, sus mínimos se determinaron en diciembre, cuando ocurrió un evento moderado de surgencia (Tabla 1).

Las especies restantes fueron escasas y ocasionales. Solamente en función de su presencia-ausencia, se observaron algunas variaciones en su distri- 

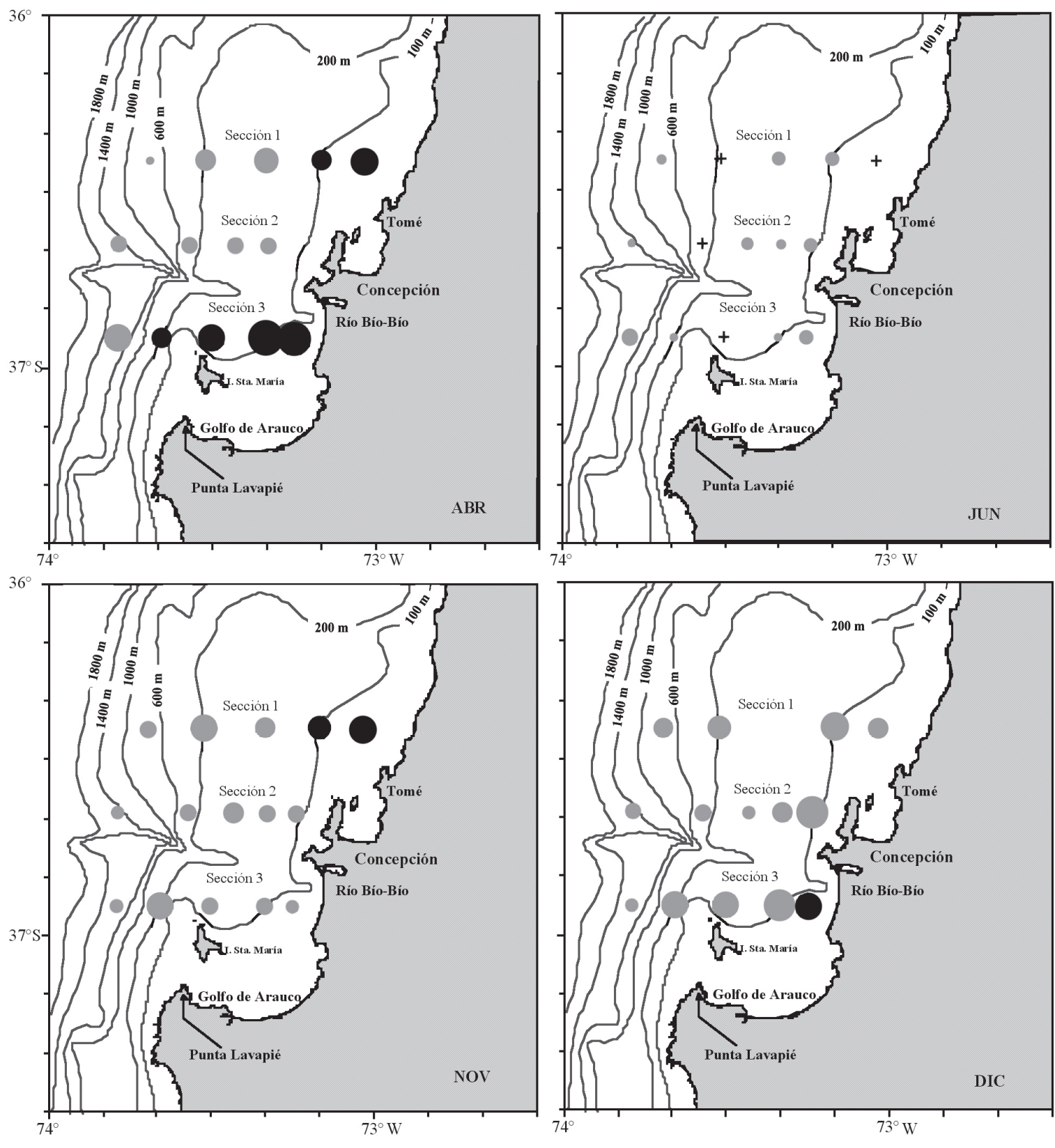

Estaciones nocturnas

Estaciones diurnas

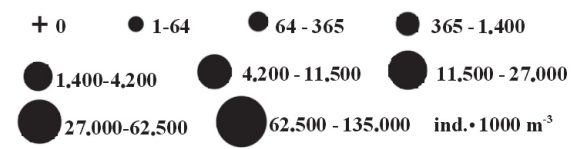

Figura 3. Distribución espacio-temporal de Euphausia mucronata frente a la costa de Concepción.

Figure 3. Spatial and temporal distribution of Euphausia mucronata off Concepción coast. 

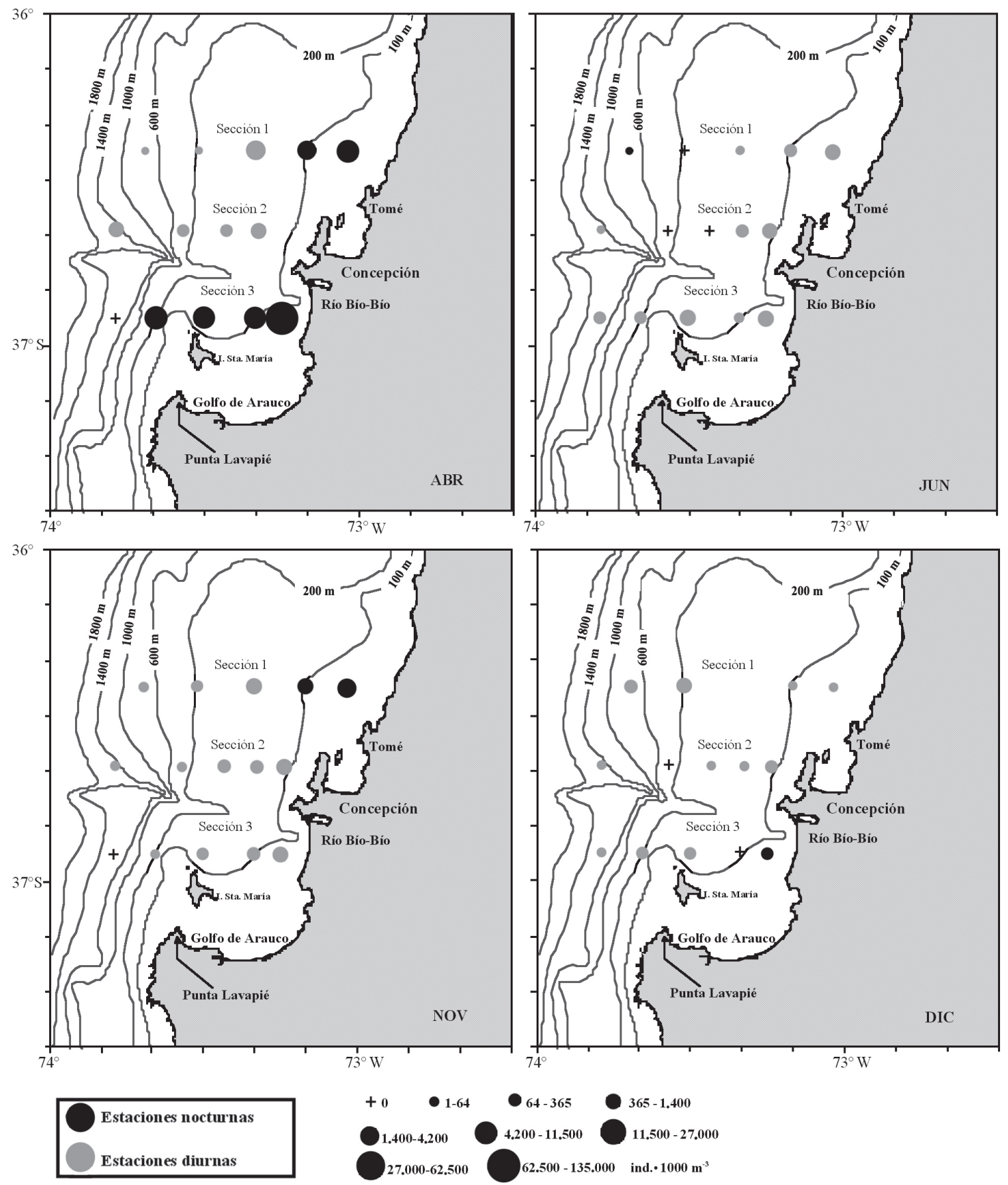

Figura 4. Distribución espacio-temporal de Nyctiphanes simplex frente a la costa de Concepción.

Figure 4. Spatial and temporal distribution of Nyctiphanes simplex off Concepción bay. 

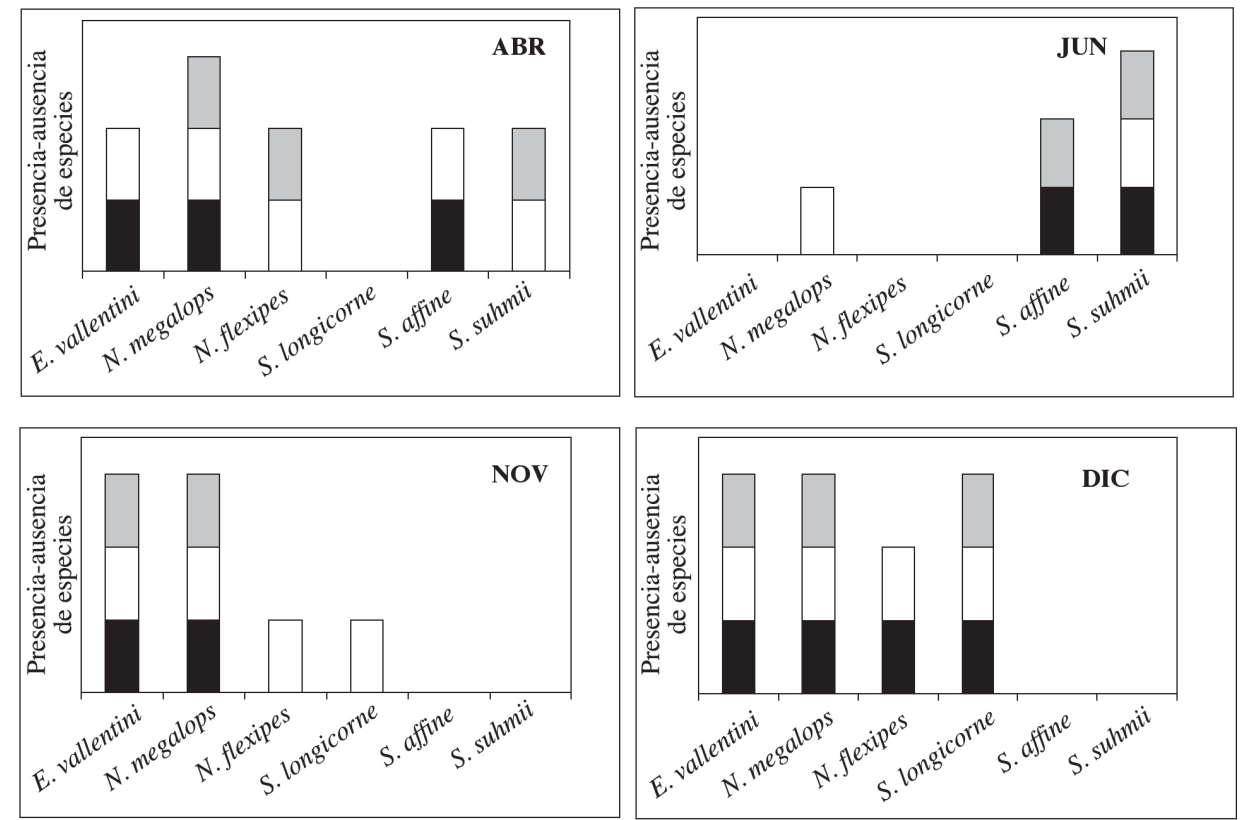

Sección 1

Sección 2

Sección 3

Figura 5. Presencia-ausencia de las especies ocasionales en la secciones oceanográficas.

Figure 5. Presence-absence of occasional species in the oceanographic sections.
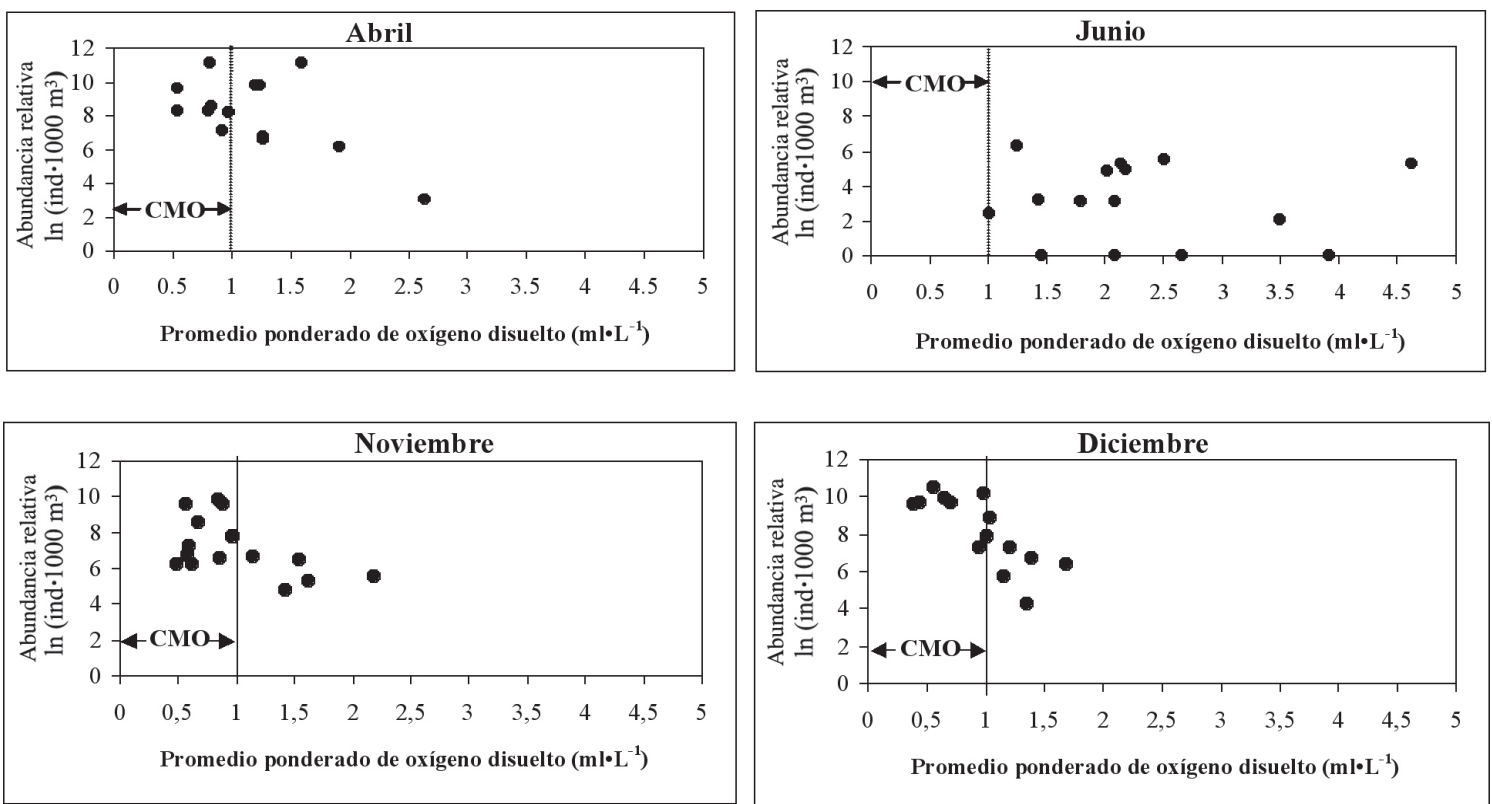

Figura 6. Variabilidad temporal de la abundancia de Euphausia mucronata con relación a la concentración de oxígeno disuelto ponderado $\left(\mathrm{ml} \cdot \mathrm{L}^{-1}\right)$ en la columna de agua. CMO: Capa de Mínimo Oxígeno.

Figure 6. Temporal variability of Euphausia mucronata abundance related to the water column weighted dissolved oxygen concentration $\left(\mathrm{ml} \cdot \mathrm{L}^{-1}\right)$. CMO: Oxygen Minimum Layer. 
bución entre los diferentes periodos analizados (Fig. 5). En abril se encontró el mayor número de especies ocasionales y en junio la menor cantidad. En diciembre se determinó una mayor cobertura geográfica de estas especies. De este conjunto de especies ocasionales, destacó la presencia de $S$. suhmii que es característica del Pacífico Central y que se capturó solo en abril y junio (Fig. 5).

\section{Euphausia mucronata y su relación con la Capa de Mínimo Oxígeno (CMO)}

Al comparar los valores ponderados de la concentración de oxígeno disuelto en la columna de agua (0-200 m) con las capturas de E. mucronata en cada crucero, se detectaron importantes correlaciones (Fig. 6). La mayor parte del año, sus mayores densidades estuvieron asociadas a bajas concentraciones de oxígeno, aunque sólo en abril y diciembre esta relación inversa fue significativa (Prueba de Pearson, $\left.\mathrm{r}_{\mathrm{abr}}=-0,62 ; \mathrm{r}_{\mathrm{dic}}=-0,79 ; \mathrm{p}<0,05\right)$. En cambio en junio, cuando la abundancia relativa fue la más baja del año, se registraron valores de oxígeno disuelto superiores a $1 \mathrm{ml} \cdot \mathrm{L}^{-1}$ (Fig. 6).

\section{DISCUSIÓN}

En el SCH los estudios sobre los eufáusidos en aguas costeras son escasos y se han centrado en la zona norte, frente a Antofagasta (Escribano et al., 2000; Fernández et al., 2002). Por lo tanto, los resultados obtenidos en esta investigación constituyen el primer aporte al conocimiento de la distribución espacio-temporal de los eufáusidos en la zona centrosur del país. La mayoría de las especies encontradas frente a la costa de Concepción había sido registradas previamente en aguas costeras y oceánicas del Pacífico suroriental (Brinton, 1962; Antezana, 1978, 1981; Escribano et al., 2000; Ulloa et al., 2000; Fernández et al., 2002), a excepción de S. suhmii que se distribuye comúnmente en la región central del Pacífico, entre los $10-40^{\circ} \mathrm{N}$ y $10-40^{\circ} \mathrm{S}$ (Gibbons et al., 1999) y que se registra por primera vez para el SCH.

La variabilidad en la abundancia de los eufáusidos en los distintos periodos de muestreo, se debería principalmente a la estacionalidad en los patrones de abundancia y distribución de juveniles/ adultos de E. mucronata, que constituyeron el $76 \%$ del total de ejemplares. Su alta abundancia puede explicar incluso la biomasa total del zooplancton, ya que sus fluctuaciones estacionales son coinciden- tes con el máximo otoñal y mínimo invernal de biomasa registrados en esta misma área (Palma, 1994). Según Antezana (1978, 2002a), su abundancia constituye el $46 \%$ de la biomasa zooplanctónica entre $\operatorname{los} 10^{\circ}$ y $39^{\circ} \mathrm{S}$ en el SCH.

E. mucronata es una especie endémica del SCH, de extraordinaria abundancia (Brinton, 1962), capaz de formar agregaciones comparables a las de $E$. superba en aguas antárticas (Gunther, 1936). Se distribuye a los $5^{\circ}-46^{\circ} \mathrm{S}$ y forma densas concentraciones entre $\operatorname{los} 30^{\circ}$ y $43^{\circ} \mathrm{S}$, particularmente frente a Talcahuano, donde alcanza máximos superiores a $30 \mathrm{ind} \cdot \mathrm{m}^{-3}$ en una franja restringida a 60 millas de la costa (Antezana, 1970). La abundancia de esta especie en el SCH, permite sustentar una parte significativa de la dieta de numerosos recursos marinos, como ballenas, y peces pelágicos y demersales (Antezana, 1970, 1978).

Las agregaciones de E. mucronata frente a la costa de Concepción fueron muy superiores a las registradas en la zona norte de Chile. En efecto, en diciembre de 1991 se determinaron concentraciones de hasta 146 ind $\cdot \mathrm{m}^{-3}$, en comparación con los 20 ind $\cdot \mathrm{m}^{-3}$ registrados en diciembre de 1996 frente a Antofagasta (Escribano et al., 2000). En esta última localidad, González et al. (2000) determinaron concentraciones de E. mucronata y E. eximia de 54 ind $\cdot \mathrm{m}^{-3}$ en periodos no-El Niño (enero 1997) y de 4 ind $\cdot \mathrm{m}^{-3}$ en período El Niño (enero 1998).

A pesar que se detectaron diferencias significativas entre las capturas diurnas y nocturnas de $E$. mucronata, su patrón de distribución estacional parece más relacionado con las fluctuaciones estacionales de la AESS, tanto en profundidad como en distancia de la costa, que con la hora de pesca. En efecto, en invierno, cuando predomina el viento N, la AESS se encuentra más allá del quiebre de la plataforma, alrededor de los 300-340 m de profundidad (Sobarzo et al., 1993). En cambio, durante el periodo más cálido que se extiende desde mediados de primavera a principios de otoño, predominan los vientos del S-SW y la AESS se acerca a la costa, detectándose al interior de las bahías (Ahumada et al., 1983; Arcos et al., 1987). Así, este patrón en la circulación, puede estar afectando la distribución de esta especie frente a la costa de Concepción. En efecto, en el área de estudio se detectó una relación inversa entre E. mucronata y la concentración de oxígeno disuelto (Fig. 6), estimándose que los máximos de abundancia estuvieron asociados a bajas concentraciones de oxígeno disuelto $\left(<1 \mathrm{ml} \cdot \mathrm{L}^{-1}\right)$, 
propios del AESS. Particularmente, los máximos de E. mucronata en primavera y principios de otoño, estuvieron correlacionados a la CMO. En consecuencia, la abundancia de esta especie sobre la plataforma continental está fuertemente influenciada por la advección estacional del AESS hacia la zona costera.

La existencia de una relación inversa entre $E$. mucronata y la concentración de oxígeno disuelto ha sido mencionada por diversos autores para el SCH (Teal \& Carey, 1967; Antezana, 1978, 2002a, 2002b; Escribano et al., 2000; Ulloa et al., 2000). La presencia de E. mucronata en la CMO, juega un importante rol en el ecosistema, ya que es un activo filtrador y es presa de numerosos peces pelágicos y demersales (Antezana, 1978; 2002a). Adaptaciones morfológicas y/o fisiológicas, como una mayor superficie branquial, activa natación y capacidad de respirar eficazmente bajo condiciones anóxicas por periodos prolongados, les permiten a los juveniles y adultos introducirse en la CMO durante el día (Antezana, 2002a, 2002b). Antezana (2002b) sugiere que este metabolismo activo dentro de la CMO, le permitiría evadir a los predadores y alimentarse con un mínimo de competencia de fuentes complementarias o suplementarias en un entorno inhóspito para otras especies.

En la zona de Concepción, también se ha señalado la asociación entre el copépodo Rhincalanus nasutus y la CMO, donde ocurren intrusiones de esta especie oceánica favorecidas por advección costera del AESS, durante épocas de surgencia y alta productividad fitoplanctónica (Castro et al., 1993). Estos autores sugieren que la migración vertical y ontogénica de $R$. nasutus, en conjunto con el patrón de circulación existente, favorecen el mantenimiento de una población reproductiva en aguas costeras. Así, al igual que para este copépodo, este acoplamiento físico-biológico podría explicar las grandes densidades costeras de E. mucronata en épocas de surgencia.

$N$. simplex, segunda en abundancia después de E. mucronata, también contribuyó a las fluctuaciones espacio-temporales, particularmente en abril cuando ocurrieron sus máximos (Fig. 4). Esta especie de amplia distribución en el Pacífico oriental, se distribuye en el Sistema de la Corriente de California y Golfo de California (Brinton, 1962), y desde las Islas Galápagos hasta los $38^{\circ} \mathrm{S}$ (Antezana, 1978, 1981). Su patrón espacio-temporal observado frente a Concepción, es similar al registrado en aguas costeras ecuatorianas (Cornejo, 1976), donde sus máximos ocurren en mayo y sus mínimos en diciembre. En Baja California $\left(23^{\circ}-29^{\circ} \mathrm{N}\right)$, Gómez (1995) encontró sus máximos en aguas costeras, sugiriendo que su distribución nerítica sería una adaptación a condiciones de surgencia, evitando así su advección hacia aguas oceánicas. Lavaniegos (1994) también en Baja California $\left(27^{\circ}-30^{\circ} \mathrm{N}\right)$, señala que la migración vertical de los individuos maduros evitaría su advección hacia aguas oceánicas durante eventos de surgencia. En esta misma zona, se ha señalado que Bahía Vizcaíno constituye un área de retención de adultos de $N$. simplex, que se alimentan preferentemente en invierno y se reproducen en otoño, patrón asociado al desarrollo de un fuerte giro anticiclónico al término de la época de surgencia (Lavaniegos, 1994, 1995; De Silva et al., 2002). Similares resultados se han detectado en otras bahías de Baja California, donde una combinación entre estabilidad de la columna de agua y circulación semi-cerrada, reduce las pérdidas advectivas y favorece la existencia de altas concentraciones de alimento (De Silva \& Palomares, 1998). La presencia del género Nyctiphanes en áreas de surgencia, parece un patrón común de este género, ya que Nyctiphanes couchii (Le Roux, 1973) y N. australis (Bradford \& Chapman, 1988) tienen comportamientos similares.

Las especies restantes fueron ocasionales frente a Concepción, aunque su presencia puede ser indicadora de condiciones hidrodinámicas específicas. Así, la presencia de E. vallentini, especie común en aguas subantárticas y frecuente en aguas del $\mathrm{SCH}$ a los $37^{\circ}-42^{\circ} \mathrm{S}$ (Antezana 1978, 1981; Antezana et al., 1997), se debería a un acoplamiento físicobiológico en esta área, relacionado con un fuerte flujo costero hacia el norte asociado a eventos de surgencia, sobre todo en noviembre y diciembre cuando presentó una mayor cobertura geográfica. Asimismo, aunque este es el primer hallazgo de $S$. suhmii en el SCH, esta especie ya había sido previamente registrada en aguas oceánicas de latitudes cercanas $\left(35^{\circ} \mathrm{S}\right)$ (Brinton, 1962; Mauchline \& Fisher, 1969). Es posible que su presencia frente a Concepción, se debería a un acercamiento a la costa de aguas provenientes del Pacífico central.

Según Siegel (2000), existen varios factores que pueden controlar los cambios temporales y espaciales en los eufáusidos, como factores físicos que condicionan el medio ambiente (temperatura, circulación), factores poblacionales (éxito de desove, mortalidad y reclutamiento), y factores de su comporta- 
miento (migraciones verticales y horizontales). Frente a Concepción, las condiciones oceanográficas poseen un alto dinamismo (Djurfeldt, 1989; Cáceres \& Arcos, 1991; Mesías et al., 2001), reflejado en el desarrollo permanente de procesos de surgencia, hundimiento, remolinos, cambios en la circulación y aportes del río Bío-Bío. Estas condiciones explicarían las densas agregaciones de E. mucronata y $N$. simplex (Figs. 3 y 4), registradas en abril y diciembre en el golfo de Arauco. Estos incrementos en el sector sur, serían aún más favorecidos por el patrón de circulación descrito por Salinas (1992), caracterizado por un fuerte flujo hacia el sur de la corriente subsuperficial y la presencia del AESS en el golfo de Arauco (Silva, 1993). En cambio, las agregaciones registradas en el sector norte se deberían a la exportación de poblaciones, desde las productivas aguas de la bahía de Concepción (Mesías \& Salinas, 1986; Arcos et al., 1987; Ahumada et al., 1983, 1991). Los mínimos de abundancia registrados en junio se deberían a la mayor estabilidad de la columna de agua, y en menor grado, al aumento del caudal del río Bío-Bío, cuyas fluctuaciones pueden afectar la biomasa zooplanctónica en el golfo de Arauco.

La variabilidad espacio-temporal de estos organismos, podría también estar influenciada por otros factores relacionados con el comportamiento de los organismos. Al respecto, el documentado patrón migratorio de estas especies (Brinton, 1967; Lavaniegos, 1996; Gómez \& Robinson, 1997; Antezana, 2000a; Escribano et al., 2000; Fernández et al., 2002), podría determinar en parte, un aumento en su abundancia durante la noche, en las estaciones más costeras del golfo de Arauco (Figs. 3 y 4). Sus desplazamientos en la columna de agua, generan un mecanismo que explica su desarrollo y permanencia dentro del área de estudio durante todo el año, tal como ocurre con E. lucens en el sistema de surgencia del sur de Benguela. En esta localidad, estadios más avanzados y adultos, por su amplia capacidad migratoria son transportados al norte y fuera de la costa (transporte de Ekman), por un flujo costero en capas cercanas a superficie y luego son transportadas hacia el sur y hacia la costa a través de una contracorriente más profunda hacia el polo (Pillar \& Stuart, 1988; Pillar et al., 1989). Un caso análogo se registra en la zona norte de la Corriente de Benguela, donde individuos maduros de Nyctiphanes capensis presentan un comportamiento migratorio nictimeral para reducir pérdidas por advección (Barange \& Pillar, 1992). Al parecer, el patrón de circulación en zonas de surgencia permite no sólo una alta producción primaria, sino también la presencia de algunas especies, que dependiendo de su capacidad natatoria pueden utilizar este sistema dinámico como un eficaz mecanismo de transporte y retención para varios zooplancteres, como Calanus marshallae, Calanoides carinatus, Rhincalanus nasutus, E. lucens y N. simplex, que son comunes en áreas de surgencia en los sistemas de la Corrientes de California, Benguela o Humboldt (Peterson et al., 1979; Verheye \& Fields, 1992; Pillar et al., 1989; Lavaniegos, 1994).

Asimismo, aunque en este trabajo no se consideraron los estadios tempranos de estas especies, es posible que su variabilidad temporal sea afectada por su ciclo de vida. Las menores abundancias de juveniles y adultos detectadas en primavera, se deberían a un incremento larval en esta época del año, que estaría reemplazando parte de la población adulta registrada en esta área. Lo anterior, estaría favorecido por un aumento en la disponibilidad trófica, asociado a moderados eventos de surgencia detectados en noviembre (Silva, 1993).

Los resultados obtenidos en conjunto con las características hidrográficas de la zona de Concepción, sugieren que la comunidad de eufáusidos, representada esencialmente por E. mucronata, fundamenta su variabilidad estacional y espacial en el comportamiento coordinado de dos tipos: uno pasivo, debido a su asociación con masas de agua que los transportan, dispersan o retienen; y uno activo, por la capacidad intrínseca de estadios de desarrollo y adultos para desplazarse a cortas o grandes distancias en la columna de agua. Es posible que este acoplamiento dinámico sea el principal responsable de la alta productividad en muchos ecosistemas marinos de surgencia costera, similares al Sistema de la Corriente de Humboldt.

\section{AGRADECIMIENTOS}

Los autores agradecen al Dr. Tarsicio Antezana de la Universidad de Concepción, por su colaboración en la identificación de algunos eufáusidos así como sus valiosos comentarios que sirvieron de apoyo para la elaboración de este manuscrito, y al Prof. Nelson Silva de la Pontificia Universidad Católica de Valparaíso, que facilitó los datos de oxígeno disuelto utilizados en este trabajo. 


\section{REFERENCIAS}

Ahumada, R., A. Rudolph \& V. Martínez. 1983. Circulation and fertility of waters in Concepción Bay. Estuar. Coast. Shelf Sci., 16: 95-105.

Ahumada, R., P. Matrai \& N. Silva. 1991. Phytoplankton biomass distribution and relationship to nutrient enrichment during an upwelling event off Concepción Bay Chile. Bol. Soc. Biol. Concepción, 62: 7-19.

Antezana, T. 1970. Eufáusidos de la costa de Chile. Su rol en la economía del mar. Rev. Biol. Mar., 14(2): 19-27.

Antezana, T. 1978. Distribution of euphausiids in the Chile-Perú Current with particular reference to the endemic Euphausia mucronata and the Oxygen Minimum Layer. Ph. D. Dissertation, San Diego. Scripps Institution of Oceanography, University of California, San Diego, 465 pp.

Antezana, T. 1981. Zoogeography of euphausiids of the South Eastern Pacific Ocean. En: UNESCO (ed.). Memorias del Seminario sobre Indicadores Biológicos del Plancton. Oficina Regional de Ciencia y Tecnología de la UNESCO para América Latina y el Caribe, ROSTLAC, Montevideo, 5-23 pp.

Antezana, T. 2002a. Vertical distribution and diel migration of Euphausia mucronata in the oxygen minumum layer of the Humboldt Current. En: J. Farber (ed.). Oceanography of the Eastern Pacific II. CICESE, Ensenada, pp. 13-28.

Antezana, T. 2002b. Adaptative migratory behavior of Euphausia mucronata in the oxygen minumum layer of the Humboldt Current. En: J. Farber (ed.). Oceanography of the Eastern Pacific II. CICESE, Ensenada, pp. 29-40.

Antezana, T. \& E. Brinton. 1981. Euphausiacea. En: D. Boltovskoy (ed.). Atlas del Zooplancton del Atlántico Sudoccidental y métodos de trabajo con el zooplancton marino. Instituto Nacional de Investigación y Desarrollo Pesquero, Mar del Plata, pp. 681-698.

Antezana, T., G. Benasi, G. Costanzo, N. Crescenti, I. Ferrari, E. Ghirardelli, A. Granata, L. Guglielmo \& K. Mckenzie. 1997. Atlas of marine zooplankton Straits of Magellan. Amphipods, Euphausiids, Mysids, Ostracods and Chaetognaths. Spinger Verlag, Berlin, 275 pp.
Arcos, D., S. Núñez, L. Castro \& N. Navarro. 1987. Variabilidad vertical de clorofila $a$ en un área de surgencia frente a Chile central. Invest. Pesq., Chile, 34: 47-55.

Barange, M. \& S. Pillar. 1992. Cross-shelf circulation, zonation and maintenance mechanisms of Nyctiphanes capensis and Euphausia hanseni (Euphausiacea) in the northern Benguela upwelling system. J. Cont. Shelf Res., 12(9): 1027-1042.

Bradford, J. \& B. Chapman. 1988. Nyctiphanes australis (Euphausiacea) and an upwelling plume in western Cook Strait, New Zealand. N. Z. J. Mar. Freshwater Res., 22: 237-247.

Brinton, E. 1962. The distribution of Pacific euphausiids. Bull. Scripps Inst. Oceanogr. Univ., Calif., 8(2): 51-270.

Brinton, E. 1967. Vertical migration and avoidance capability of euphausiids in the California Current. Limnol. Oceanogr., 12: 451-483.

Brinton, E. \& J. Reid. 1986. On the effect of interannual variations in circulation and temperature upon euphausiids of the California Current. En: A.C. Pierrot-Bults, S. Van der Spoel, B. Zahuranec \& R. Johnson (eds.). A pelagic biogeography. UNESCO Tech. Pap., Mar. Sci., Paris, 49: 25-34.

Cáceres, M. \& D. Arcos. 1991. Variabilidad en la estructura espacio-temporal de un área de surgencia frente a la costa de Concepción, Chile. Invest. Pesq., Chile, 36: 27-38.

Castro, L., P. Bernal \& V. Troncoso. 1993. Coastal intrusion of copepods: mechanisms and consequences on the population biology of Rhincalanus nasutus. J. Plankton Res., 15: 501515.

Cornejo, M. 1976. Estudio preliminar de los eufáusidos del mar ecuatoriano. Bol. Inst. Oceanogr. Ecuador, 3(1): 1-46.

De Silva, R. \& R. Palomares. 1998. Unusual larval growth production of Nyctiphanes simplex in Bahía de La Paz, Baja California Sur, México. J. Crust. Biol., 18: 490-498.

De Silva, R., R. Palomares, A. Martínez \& M. Carballido. 2002. Standing stock of Nyctiphanes simplex in the southern region of the California Current System. J. Plankton Res., 24: 1057-1066. 
Djurfeldt, L. 1989. Circulation and mixing in a coastal upwelling embayment; Gulf of Arauco, Chile. J. Cont. Shelf Res., 9(11): 1003-1016.

Escribano, R., V. Marín \& C. Irribarren. 2000. Distribution of Euphausia mucronata at the upwelling area of Peninsula Mejillones, northern Chile: the influence of the oxygen minimum layer. Sci. Mar., 64(1): 69-77.

Fernández, D., R. Escribano \& P. Hidalgo. 2002. Distribución de eufáusidos en el sistema de surgencia frente a la península de Mejillones $\left(23^{\circ} \mathrm{S}\right)$ asociada a condiciones previas y durante El Niño 1997-98. Invest. Mar., Valparaíso, 30(1): 25-43.

Frontier, S. 1980. Métodos de análisis rápido en muestras planctónicas. Acta Oceanogr. Pacífico, 1(1): 137-145.

Gibbons, M., M. Barange \& L. Hutchings. 1995. Zoogeography and diversity of euphausiids around Southern Africa. Mar. Biol., 123: 257-268.

Gibbons, M.J., V. Spiridinov \& G. Tarling. 1999. Euphausiacea. En: D. Boltovskoy (ed.). South Atlantic Zooplankton. Backhyus Publishers, Leiden, pp. 1241-1279.

Gómez, J. 1995. Distribution patterns, abundance and population dynamics of the euphausiids Nyctiphanes simplex and Euphausia eximia off the west coast of Baja California, México. Mar. Ecol. Prog. Ser., 119: 63-76.

Gómez, J. \& C. Robinson. 1997. Circadian biomass and abundance changes of five euphausiid species along the west coast of Baja California Mexico, December 1993. Sci. Mar., 61(1): 27-35.

González, H., M. Sobarzo, D. Figueroa \& E. Nöthig. 2000. Composition, biomass and potential grazing impact of the crustacean and pelagic tunicates in the northern Humboldt Current area off Chile: differences between El Niño and non-El Niño years. Mar. Ecol. Prog. Ser., 195: 201-220.

Gopalakrishnan, K. 1975. Biology and taxonomy of the genus Nematoscelis (Crustacea, Euphausiacea). US. Fish. Bull., 73(4): 797-814.

Gunther, E. 1936. A report on the oceanographical investigations in the Peru Coastal Current. Discovery Rep., 13: 107-276.

Lavaniegos, B. 1994. Dispersion and development patterns in larvae of Nyctiphanes simplex
(Euphausiacea) in the upwelling region off Baja California. Mar. Ecol. Prog. Ser., 106: 207-225.

Lavaniegos, B. 1995. Production of the euphausiid Nyctiphanes simplex in Viscaíno Bay, Western Baja California. J. Crust. Biol., 15(3): 444-453.

Le Roux, A. 1973. Observation sur le developpement larvaire de Nyctiphanes couchii (Crustacea: Euphausiacea) au laboratoire. Mar. Biol., 22: 159166.

Mauchline, J. \& L. Fisher. 1969. The biology of euphausiids. Adv. Mar. Biol., 7: 1-454.

Mesías, J. \& S. Salinas. 1986. Corrientes en la bahía de Concepción, Chile. Biol. Pesq., 15: 55-62.

Mesías, J., R. Matano \& T. Strub. 2001. A numerical study of the upwelling circulation off central Chile. J. Geophys. Res., 106(C9): 19611-19623.

Palma, S. 1994. Distribución y abundancia de larvas de langostino colorado Pleuroncodes monodon frente a la costa de Concepción, Chile. Invest. Mar., Valparaíso, 22: 13-29.

Peterson, W., C. Miller \& A. Hutchinson. 1979. Zonation and maintenance of copepod populations in the Oregon upwelling zone. Deep-Sea Res., 26A: 467-493.

Peterson, W., D. Arcos, G. McManus, H. Dam, D. Bellantoni, T. Johnson \& P. Tiselius. 1988. The nearshore zone during coastal upwelling: daily variability and coupling between primary and secondary production off Central Chile. Progr. Oceanogr., 20: 1-40.

Pillar, S. \& V. Stuart. 1988. Population structure, reproductive biology and maintenance of Euphausia lucens in the southern Benguela Current. J. Plankton Res., 10(6): 1083-1098.

Pillar, S., D. Armstrong \& L. Hutchings. 1989. Vertical migration, dispersal and transport of Euphausia lucens in the southern Benguela Current. Mar. Ecol. Prog. Ser., 53: 179-190.

Salinas, S. 1992. Corrientes sobre la plataforma continental de Talcahuano. Estud. Doc., Univ. Católica Valparaíso, 22/92: 35 pp.

Siegel, V. 2000. Krill (Euphausiacea) demography and variability in abundance and distribution. Can. J. Fish. Aquat. Sci., 57(Suppl. 3): 151-167.

Silva, N. 1993. Condiciones oceanográficas y 
sedimentológicas frente a la bahía de Concepción durante los cruceros de prospección del langostino colorado (Pleuroncodes monodon) (marzo a diciembre de 1991). Estud. Doc., Univ. Católica de Valparaíso, 6/93: 51 pp.

Sobarzo, M., E. Sansore, A. De Maio, D. Arcos, M. Salamanca \& J. Henríquez. 1993. Variabilidad espacio-temporal de la estructura hidrográfica de las aguas del Golfo de Arauco. En: F. Faranda \& O. Parra (eds.). Oceanografía física del golfo de Arauco. Universidad de Concepción. Serie Monográficas Científicas, 4: 60 pp.

Teal, J. \& F. Carey. 1967. Respiration of a euphausiid from the oxygen minumum layer. Limnol. Oceanogr., 12: 548-550.

Recibido: 15 junio 2003; Aceptado: 19 marzo 2004
Ulloa, R., S. Palma, L. Linacre \& N. Silva. 2000. Seasonal changes in the bathymetric distribution of siphonophores, chaetognaths and euphausiids associated to water masses off of Valparaiso, Chile (Southeast Pacific). En: J. Farber (ed.). Oceanography of the Eastern Pacific I. CICESE, Ensenada, pp. 72-83.

Verheye, H. \& J. Field. 1992. Vertical distribution and diel vertical migration of Calanoides carinatus (Krøyer, 1849) development stages in the Southern Benguela upwelling region. J. Exp. Mar. Biol. Ecol., 158: 122-140. 